\title{
Selective Absorption
}

This content has been downloaded from IOPscience. Please scroll down to see the full text. 1875 Proc. Phys. Soc. London 2110

(http://iopscience.iop.org/1478-7814/2/1/310)

View the table of contents for this issue, or go to the journal homepage for more

Download details:

IP Address: 130.179.16.201

This content was downloaded on 09/09/2015 at $14: 36$

Please note that terms and conditions apply. 
13. This conclusion can be directly proved by recurring to the earlier experiments (fig. 2) and introducing across the circuit a wire of suitable resistance to short-circuit the extracurrent so that it may complete its route without having to uverleap the break at the key. This is shown in diagram in fig. 6 , where $\mathrm{R}$ is a box of resistance-coils introduced between $a$ and $a^{\prime}$. If the wire of which the electromagnet $\mathrm{E}$ is made be stout, its resistance will be a mere trifle, and a resistance of 3 or 4 ohms at $R$ will be sufficient. In some cases a drop of pure water between the points of contact at $K$ has also the result of obviating the extra-current spark. And in every case, when the conditions are so arranged as to obviate the appearance, on breaking contact, of the extra-current spark at $K$, no earth-wire, nor any conductor, will draw a spark from the electromagnet-core-a decisive proof that the sparks so drawn were due to induction by the statical charge of the accumulating extra-current, and to that alone.

\section{Selective Absorption. By William Acrroyd.}

AT present much attention is being paid to absorptionspectra for technological as well as purely scientific reasons; but in the absence of classification and of a general theory accounting for the facts observed, the whole subject is surrounded by a haze which it were well for progress to dispel. Much perplexity and not a few mistakes have arisen from the confusing of two kinds of absorption, or at least two different manifestations of the same phenomenon, which hitherto have not been sufficiently contradistinguished. These we propose to classify on the basis of the following typical experiments.

1. Light is transmitted through a thin layer of potassic dichromate at the normal temperature, and again at a little below its fusing-point. We obtain spectra approximately represented by Nos. 1 \& 2 . It will presently be shown that this increase of absorption at the higher temperature is the result of structural alterations in the dichromate. We shall therefore call this kind of absorption structural absorption.

2. A glass cell, 15 centims. $\times 3$ centims., contains cupric 
sulphate in solution. Iight sent through the 3-centims. direction gives spectrum No. 3, whilst light transmitted through the 15-centims. direction gives spectrum No 4 . We have here increase of absorption without any structural alterations in the absorbing medium; for the cupric sulphate remains the same during the two observations, and the temperature is unaltered. The only difference is one of extent of medium. For this kind of absorption we propose, at the suggestion of Dr. Guthrie, the term transverse absorption.

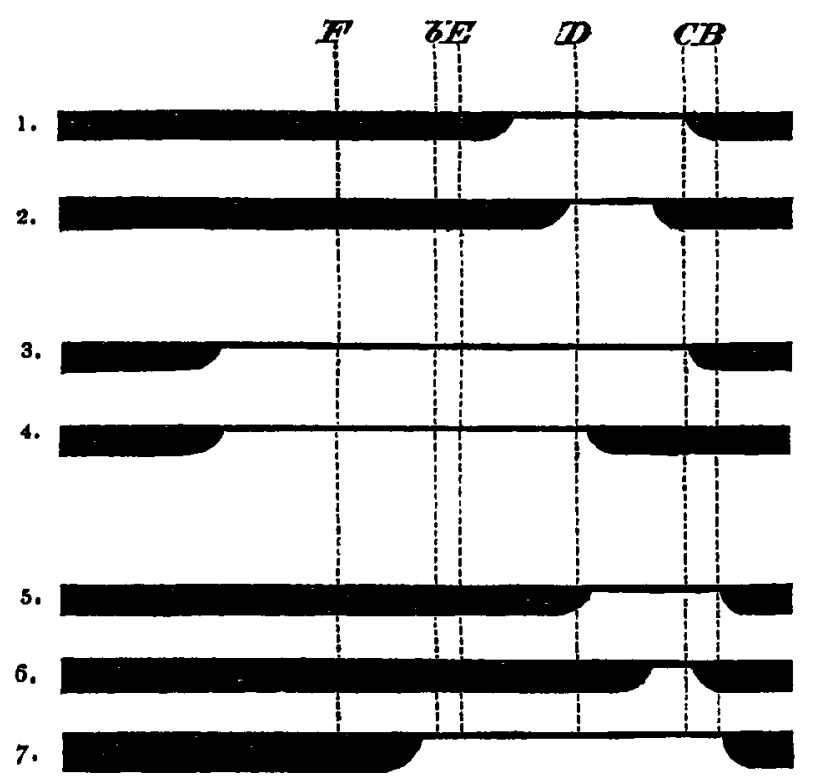

Professor Gladstone*, when speaking of the action of heat on coloured solutions, thus tacitly refers to the two kinds of absorption:- "The elevation of temperature seems merely to heighten the absorbent power of the dissolved salt, so that light absorbed by a certain quantity of the heated solution is the same as would have been absorbed by a larger quantity of the same solution if cold." Notwithstanding this, the two kinds of absorption seem not to have been formally separated; and the mixed inferences from one and the other have naturally produced a confusion in the minds of students which has made selective absorption a subject little understood. We shall confine our observations to structural absorption.

* Phil. Mag. [IV.] vol. xiv. p. 423. 
Stmictural Absorption.-Alteration of structural absorption upon elevation of temperature is, as a rule, obvious to the eye as a change of colour. This colour-change, for which $I$ have elsewhere proposed the name of metachromatism, has been studied by Schönbein, Gladstone, Houston and Thompson, and, lastly, by myself. Many theories have been offered to account for the phenomenon; these were discussed in my paper on Metachromatism*, read before the Chemical Society on the 3rd of February last.

If a little oxide of zinc be heated strongly on a piece of white porcelain, it changes from white to orange and yellow. Chromate of lead treated similarly changes from orange to deep orange, brick-red, and black-red. These bodies quickly regain their original colour with a return of the normal temperature; hence one might suppose that the chromate of lead, if cooled far below the normal temperature, would acquire in succession the yellow and white of the zinc oxide, thus (in the order of cooling)-black-red, brick-red, deep orange, orange, yellow, and white.

Such a sequence of colour-change is exhibited by nitric peroxide ; a ruddy vapour at the normal temperature, it becomes orange, yellow, and below zero a colourless liquid. From a study of such metachromatic facts and their sequence we have arrived at the following scale of change:-

Metachromatic Scale.

\begin{tabular}{|l|} 
Black \\
Heating or \\
expanding.
\end{tabular}

Cooling or contracting.

The scale may be illustrated by heating the following anhydrous bodies on white porcelain. Zinc oxide changes from white to yellow and orange; metaborate of copper from blue to green and yellowish green; baric chromate from yellow to orange; mercuric oxide from orange-yellow to orange, red,

- Chemical News, vol. xxxiv. pp. 76, 77. 
and brown ; potassic dichromate, red to dark red; trimanganic tetroxide, reddish brown to nearly black; and cuprous oxide glass from scarlet to dark scarlet and black.

Respecting this colour-change, we make out:-(1) that it arises from increased structural absorption with elevation of temperature, the more refrangible increment increasing at a greater rate than the less refrangible; and (2) that the only necessary concomitant is alteration of atomic potentiality-a change from the white towards the black end of the metachromatic scale signifying atomic recession, and a change from the black towards the white end, atomic approach.

(1) In support of this we must refer to the bichromate experiment. The change from red to dark red viewed spectroscopically gives spectra Nos. 1 and 2.

(2) To arrive at the approximate cause, I hare heated colourchanging bodies in various media, as nitrogen, carbonic anhydride, and hydrogen, the result of which has been to show that the surrounding medium has nothing to do with metachromatism. The phenomenon is exhibited by all three states of matter-by solids (amorphous and crystalline), by liquids, and by gases near their liquefying-points. By a process of elimination it may readily be seen that the phenomenon is due to the reception of energy from the heating agent. Now of this transferred kinetic energy a portion is converted into energy of position. In the metachrome or colour-changing body we have therefore-

i. Kinetic energy, and the complementary.

ii. Potential energy. This energy of position is acquired by a. The overcoming of cobesion (molecular recession or molar expansion), as, e.g., in the conversion of ice into water, and water into steam, work which is accompanied by a change of density.

$\beta$. The overcoming of chemical attraction (atomic recession or molecular expansion), which finally ends in decomposition, as, e.g., in the resolution of $\mathrm{PtCl}_{4}$ into $\mathrm{PtCl}_{2}$ and $\mathrm{Cl}_{2}$.

I. Now metachromatism is not confined to the coloured bodies one meets with in the laboratory; for many anhydrous silicates exhibit colour-change, and in some the change is permanent after the metachrome has been subjected to a high 
temperature. This is shown in the following Table* in each example save that of olivine.

Density before
ignition.

After ignition.

1. Olivine .... Pistachio-green .. $3 \cdot 389$

2. Beryl..... Straw-yellow .... 2.697

3. Topaz .... Sherry .......... 3.539

4. Zircon .... Brown ........4.515

5. $", \ldots$. Aurora-red ......4.863

Pist.-green .. 3.378

Blue ..... 2.697

Pink........ 3.533

White ...... 4.540

Colourless .. $4 \cdot 863$

If we might assume that two bodies of identical chemical composition and density have at a common temperature the same amount of kinetic energy, then it is evident that we may eliminate kinetic energy from the list of possible causes. For, to take the beryl (2), we have differences of colour (that is, of structural absorption) at a common temperature, and the same in the zircon 5, without alterations of density or chemical composition.

The behaviour of mercuric iodide is perhaps a less equivocal demonstration of this point. Examined spectroscopically at (say) $16^{\circ}$ C., a band of red light is transmitted extending from $B$ to $D$. This narrows as the temperature rises (in other words, there is an increase of structural absorption), up to about $140^{\circ} \mathrm{C}$. The band of transmitted light now suddenly widens and extends to a little beyond $b$. Spectra Nos. 5, 6, and 7 illustrate this change. After the decrease of absorption as represented by No. 7, we have the normal increase with elevation of temperature; but still at $220^{\circ} \mathrm{C}$. there is not so much light absorbed as there was at $16^{\circ} \mathrm{C}$. Now the heat or kinetic energy of a body increases with elevation of temperature; therefore the deep orange $\mathrm{HgI}_{2}$ at $220^{\circ} \mathrm{C}$. has more kinetic energy than the red modification at $16^{\circ} \mathrm{C}$.; but the latter has greater structural absorption. The rule is that increase of kinetic energy is accompanied by increased structural absorption. In the behaviour of mercuric iodide we have the opposite of this, viz. decrease of absorption with increase of kinetic energy, whence we infer that the change of absorption which characterizes metachromatism is not necessarily accompanied by change of kinetic energy.

II. Expansion by heat ( $i$. e. decrease of density) is an all

* Compiled from "Some Experiments on the Density of Garnet, \&c." (Church), Chem. Soc. Journ. rol. xvii. pp. 386 \& 415. 
but universal law so far as we at present know. There are several exceptions, however; and many of these are among the silicates. Their anomalous behaviour is, as a rule, pointed out by the colour-change, as in the case of the zircon. But there may be change of colour, as in the beryl, without alteration of density, $i . e$. without appreciable molecular approach or recession. On the other hand, we have in olivine an example of change of density (molecular recession) without a corresponding alteration of colour. More facts of the same nature might readily be adduced, from which we infer that molar expansion or contraction is not a necessary concomitant of alteration of structural absorption. We have now excluded from our list of possible concomitants $i$. and ii $\alpha$. Hence it would appear that ii $\beta$ is the only necessary concomitant-a conclusion which receives strength when we consider that the gradual increase of structural absorption in such bodies as $\mathrm{HgO}, \mathrm{PtCl}_{4}$, and $\mathrm{Cr}_{2} \mathrm{O}_{5} \mathrm{Ko}_{2}$ terminates with their resolution into lower compounds, or into their constituent elements.

Relation of Colour to Density.-An attempt was made in 1765 by Delaval to show the connexion between colour and density. In a paper read before the Royal Society, he observes * :-_ It appears from Newton's experiments that denser substances ought by their greater reflective power, in like circumstances, to reflect the less refiangible rays, and that substances of less density should reflect rays proportionably more refrangible and thereby appear of several colours in the order of their density." The seeming success of this attempt is perhaps due to the comparative nature of his facts; for he compares coloured glasses of each of the following metals in the order of their densities:-gold, red; lead, orange; silver, yellow; copper, green; iron, blue. We can readily see now the error of such a comparison; for the colour of glass must evidently depend upon the state of the metal in it; $e$. $g$. differently coloured glasses may be obtained from the different oxides of the same metal. To take an instance, whilst the cupric oxide gives a bluish-green glass, we know that the cuprous oxide gives a red one. Nevertheless it will be obvious from our foregoing observations that it is possible to establish a connexion between density and colour as due to structural absory-

- Philosophical Transactions, rul. lv. p. 10. 
tion. Not, however, until we have made out a special relation can we well proceed with certainty to establish a comparative one; nor will this special relation accord with Newton's prediction.

From the nature of metachromatism, it is probable that increase or decrease of interatomic distance will in most cases be accompanied by a corresponding alteration of molecular interspaces. Hence in allotropes we shall look for exactly the opposite of what Delaval sought to establish, viz. for cold colours with great density and warm ones with low density.

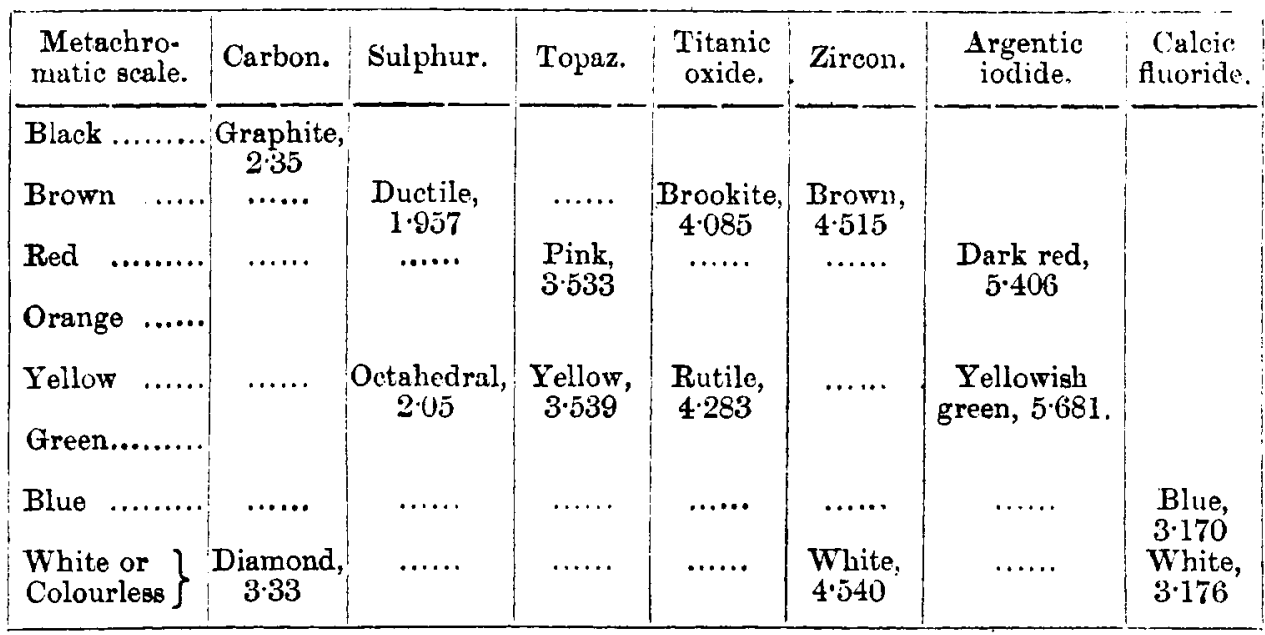

The fact of phosphorus being an exception does not militate against this relation, since it is highly probable that the other anomalies of this body will receive elucidation when studied from the metachromatic standpoint. The change in colour of mercuric iodide, which we have already referred to, indicates atomic approach; and to ascertain whether this is accompanied by a corresponding molecular motion and consequent change of density has been the object of a large number of experiments. From the peculiar difficulties encountered, however, as yet I have obtained no trustworthy results.

The next step from the special relation we have pointed out to one of a comparative nature would seem to be in an extension of the following law of colour-sequence:- "In a series of anhydrous binary compounds of the same two elements, those which have the highest amount of the basylous element exhibit the 
least structural absorption; and, vice versa , those which have the least amount of the basylous element have the most structural absorption"*.

The Five Orders of Spectra.-In the borderland between chemistry and physics, data are being accumulated which tend to make the former a dynamical science. Not the least important of these data are those which furnish us with the connexion between the different kinds of spectra. Professor Roscoe is of opiniont that there is not sufficient evidence as yet to support the sequence in the five orders proposed by Luckyer $\neq$ and now generally received; and in the light of metachromatism it may be necessary to modify this sequence. The juxtaposition of the orders constituting a class of spectra we take to be founded upon that merging of the optical characters of one order into those of another which is exhibited when the molecular changes in the radiating or absorbing body cause such optical alterations. Now confining our attention to low-temperature or absorption spectra, it is evident from what has already been said that change in structural absorption alone is available for forming a sequence of orders. Hence we consider the fifth order, as at present received, nisplaced, the unique continuous absorption being from the nature of the experiment a transwerse absorptional effect.

A sequence of orders will readily suggest itself if we keep in mind that change in absorption to which metachromatism is due. Conceive of a colourless body.capable of undergoing the whole series of change. It first reflects or transmits rays of every refrangibility; as absorption increases, the band of transmitted or reflected light gradually narrows and becomes less intense; finally the field is darkened and we get continuous absorption. What we propose, then, is shown in the following comparison, made in order that the student may have a clearer conception of the matter.

* Chemical News, vol. xxxir. p. 76.

+ Watts's Dictionary, 2nd Supplement, p. 1093.

$\ddagger$ Proceedings of the Royal Society, June 11, 1874. 
Now received.

Ist order .... Line spectre 2nd order ..... Channelled-space spectra $\}$

What we propose.

Class I.

Radiative.

Class II.

Absorptive.

1. Continuous absorption.

3rd order .... Continuous absorption at ) 2. Partial absorption, i.e. abthe blue end..........

4th order .... Continuous absorption at the red end ..........

5 th order.... Unique continuous absurption ends, or at any intermediate part of the spectrum.

3. Continuous spectrum.

Class II. 1, 2, and 3 constitute the metachromatic scale. To illustrate the bridging over of 1 and 2 no simpler experiment could be tried than the following. Take a piece of copper wire and cover with suboxide by gently heating over a Bunsen burner. Fuse the covered wire into a capillary glass tube. A scarlet glass is thus obtained which is black whilst hot, i. e. exhibits continuous absorption (1), and scarlet whilst cold, exhibiting partial absorption (2). 2 and 3 are bridged over by a host of bodies like $\mathrm{ZnO}$. When hot they, as a rule, exhibit absorption at the blue end of the spectrum (2), but when cold they are white or colourless (3).

Respecting the merging of Class I. into Class II. nothing much at present can be said, since questions are involved which require further investigation.

IX. On a Mechanical Illustration of Thermo-electric Phenomena. By Ouiver J. Lodge, B.Sc.

[With a Plate.]

THE publication of the following speculation requires an apo$\operatorname{logy}$. It is in continuation of a paper on a Mechanical Illustration of Electric Induction and Conduction, published in the November Number of the 'Philosophical Magazine;' and the sections are numbered on continuously with those in that paper; but whereas the mechanical analogies suggested in the former part of the paper are useful as mere analogies even if they do not (as I believe they do) throw some light on the actual molecular processes, the present portion contains nothing of sufficient mechanical simplicity to be of much service as mere illustration, and it therefore has little value unless the imaginary 